PREPARED FOR THE U.S. DEPARTMENT OF ENERGY, UNDER CONTRACT DE-AC02-76CH03073

PPPL-3956

PPPL-3956

UC-70

Optimized Loading for Particle-in-cell

Gyrokinetic Simulations

by

J.L.V. Lewandowski

May 2004

NM|

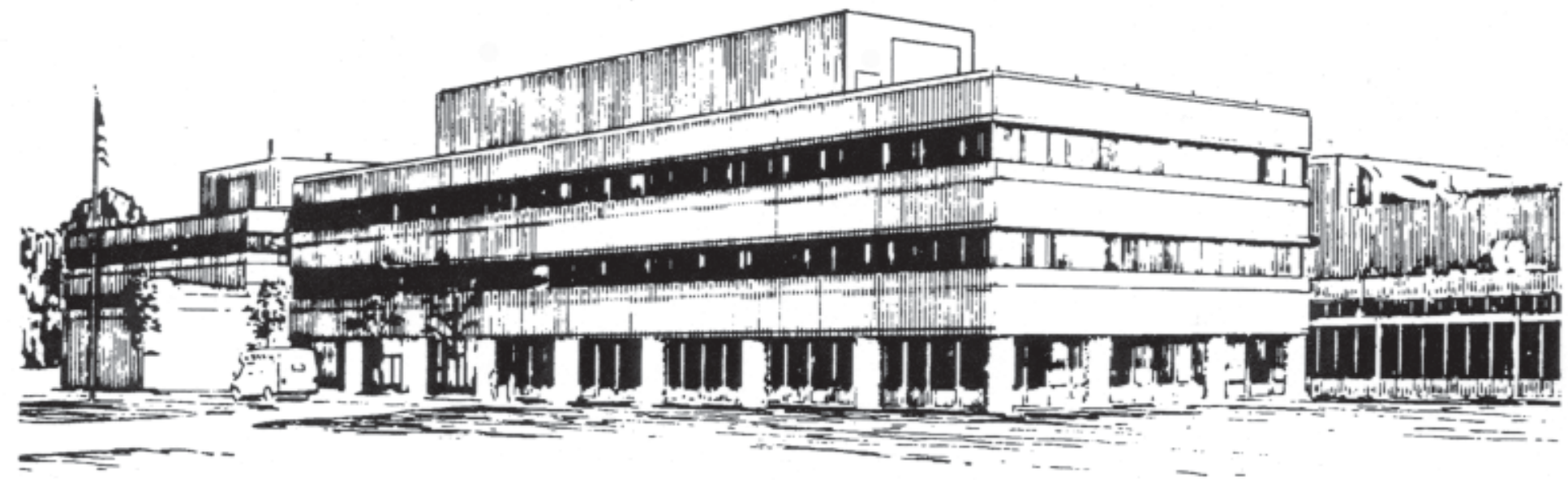

PRINCETON PLASMA PHYSICS LABORATORY PRINCETON UNIVERSITY, PRINCETON, NEW JERSEY 


\section{PPPL Reports Disclaimer}

This report was prepared as an account of work sponsored by an agency of the United States Government. Neither the United States Government nor any agency thereof, nor any of their employees, makes any warranty, express or implied, or assumes any legal liability or responsibility for the accuracy, completeness, or usefulness of any information, apparatus, product, or process disclosed, or represents that its use would not infringe privately owned rights. Reference herein to any specific commercial product, process, or service by trade name, trademark, manufacturer, or otherwise, does not necessarily constitute or imply its endorsement, recommendation, or favoring by the United States Government or any agency thereof. The views and opinions of authors expressed herein do not necessarily state or reflect those of the United States Government or any agency thereof.

\section{Availability}

This report is posted on the U.S. Department of Energy's Princeton Plasma Physics Laboratory Publications and Reports web site in Fiscal Year 2004. The home page for PPPL Reports and Publications is: http://www.pppl.gov/pub_report/

DOE and DOE Contractors can obtain copies of this report from:

U.S. Department of Energy

Office of Scientific and Technical Information

DOE Technical Information Services (DTIS)

P.O. Box 62

Oak Ridge, TN 37831

Telephone: (865) 576-8401

Fax: (865) 576-5728

Email: reports@adonis.osti.gov

This report is available to the general public from:

National Technical Information Service

U.S. Department of Commerce

5285 Port Royal Road

Springfield, VA 22161

Telephone: $1-800-553-6847$ or

(703) $605-6000$

Fax: (703) 321-8547

Internet: http://www.ntis.gov/ordering.htm 


\title{
Optimized Loading for Particle-in-cell Gyrokinetic Simulations
}

\author{
J.L.V. Lewandowski * \\ Princeton Plasma Physics Laboratory \\ Princeton University, P.O. Box 451 \\ Princeton NJ 08543 \\ USA
}

May 12, 2004

\begin{abstract}
The problem of particle loading in particle-in-cell gyrokinetic simulations is addressed using a quadratic optimization algorithm. Optimized loading in configuration space dramatically reduces the short wavelength modes in the electrostatic potential that are partly responsible for the nonconservation of total energy; further, the long wavelength modes are resolved with good accuracy. As a result, the conservation of energy for the optimized loading is much better that the conservation of energy for the random loading. The method is valid for any geometry and can be coupled to optimization algorithms in velocity space.
\end{abstract}




\section{Introduction}

The problem of microinstabilities in turbulent tokamak and stellarator plasmas has received considerable attention over the last few decades. This is mainly due to the general consensus $[1,2]$ that such short-scale, low-frequency instabilities are responsible for the so-called anomalous transport [3] that occurs across the stream lines of the confining magnetic field.

An attractive approach for the simulations of microinstabilities in complex toroidal geometries is the particle-in-cell (PIC) gyrokinetic model. In view of the inherent discreteness (through the macroparticles or markers [4]) of the PIC gyrokinetic model, the issue of energy conservation for long-time simulations is of crucial importance. There are various causes for the nonconservation of total energy in PIC gyrokinetic simulations; here are some examples: (a) the marker trajectories are integrated in time using a finite time step; (b) the computational grid in configuration space has a finite grid spacing; (c) the velocity space is not covered uniformly. In this paper, we study the problem of marker loading in configuration space. The basic idea is that an optimized loading will reduce the short-wavelength modes in the ion number density while accurately resolving the long-wavelength modes; as a result, the electrostatic potential and the local electric field that acts on the guiding centers will have fewer small-scale modes detrimental to the global energy conservation. The paper is organized as follows; in section 2, the case of one-dimensional loading based on a quadratic optimization algorithm is presented; the generalization to a two-dimensional domain is given in section 3; concluding remarks are given in section 4 .

\section{One-dimensional Optimized Loading}

Before attempting to consider the general case of optimized loading in general geometry, we present a simple one-dimensional model to illustrate the basic idea. The optimization algorithm used in this paper is based on quadratic optimization. Numerical experiments presented in this paper show that such optimization algorithm is sufficient as far as loading in configuration space is concerned; algorithms for optimized loading in velocity space sometimes do required more sophisticated techniques such as the neural network based techniques [5]. Consider a positivedefinite energy-like quantity, $E$, which depends on $N$ variables

$$
E=F\left(\theta_{1}, \theta_{2}, \cdots, \theta_{N}\right) \equiv F(\boldsymbol{\theta}),
$$

where $F$ is a nonsingular, bounded function of the variables $\theta_{i}$. Introducing a time-like variable $t$, the updating rules given by

$$
\frac{d \theta_{i}}{d t}=-\frac{\partial F}{\partial \theta_{i}} \quad(i=1,2, \cdots, N)
$$

minimize $E$; this is easily proved by using the chain rule on Eq.(1) and Eq.(2)

$$
\frac{d E}{d t}=\sum_{i=1}^{N} \frac{\partial F}{\partial \theta_{i}} \frac{d \theta_{i}}{d t}=-\sum_{i=1}^{N}\left(\frac{\partial F}{\partial \theta_{i}}\right)^{2} \leq 0 .
$$

In other words, the updating rules (2) are consistent with $d E / d t=0$ as $t \mapsto \infty$. In practise, the numerical integration of Eqs.(2) is carried out numerically since the energy-like quantity $E$ is usually a complex (nonlinear) function of the variables $\boldsymbol{\theta}$. For a given physical situation, one can conceive an infinite number of energy-like quantities $E$ with appropriate constraints (boundness 
and smoothness) on $F$. The problem is, of course, to select the proper quantity $E$.

In order to illustrate the applicability of the quadratic minimization for the case of spatial loading in PIC gyrokinetic simulations, we consider a simple one-dimensional model. The simplicity of the model is also used as a guide to determine the explicit form of the energy-like quantity $E$. In the long wavelength limit, the gyrokinetic Poisson equation is [6] (in gyrokinetic units: $\left.\rho_{s} \nabla_{\perp} \mapsto \nabla_{\perp}, e \Phi / T_{\mathrm{e}} \mapsto \Phi, \rho_{s}=c_{s} / \omega_{\mathrm{ci}}, \omega_{\mathrm{ci}}=e B /\left(m_{\mathrm{i}} c\right), c_{s}=\sqrt{T_{\mathrm{e}} / m_{\mathrm{i}}}\right)$

$$
\nabla_{\perp}^{2} \Phi=n_{\mathrm{e}}-\bar{n}_{\mathrm{i}}
$$

where $n_{\mathrm{e}}$ is the electron number density (in the limit $k_{\perp} \rho_{\mathrm{e}} \mapsto 0$, where $k_{\perp}$ is the characteristic perpendicular wavevector for ITG turbulence) and $\bar{n}_{i}$ is the ion guiding center number density which explicit form involves an integration over configuration space and velocity space

$$
\overline{n_{i}}=\int F\left(\mathbf{R}, v_{\|}, v_{\perp}\right) \delta(\mathbf{R}-\mathbf{r}+\boldsymbol{\rho}) d \mathbf{R} d \mathbf{v} .
$$

Here the position vector, $\mathbf{r}$, is related to the guiding center position vector, $\mathbf{R}$, through the relation $\mathbf{r}=\mathbf{R}+\boldsymbol{\rho}$ where $\boldsymbol{\rho}=v_{\perp} / \omega_{\mathrm{ci}}\left(\mathbf{e}_{1} \cos \varphi+\mathbf{e}_{2} \sin \varphi\right) ; \mathbf{e}_{1}$ and $\mathbf{e}_{2}$ are two orthonormal vectors that are perpendicular to the magnetic field direction at the guiding center position $\mathbf{R}$ and $\varphi$ is the gyrophase angle. The distribution function of the ion guiding centers is denoted $F$ in the above equation. Note that the real difficulty is the spatial loading of the ion guiding center positions; for the electrons, a random spatial loading is sufficient since, to a good approximation, $k_{\perp} \rho_{e} \approx 0$ (in other words, $\mathbf{R} \approx \mathbf{r}$ for the electrons). For a one-dimensional periodic system (with period $L$ ), and noting that $\bar{n}_{i} \mapsto n_{i}$ (gyroaveraging is meaningful in two-dimensional configuration space or three-dimensional configuration space), Eq.(4) becomes

$$
\frac{d^{2} \Phi}{d x^{2}}=n_{e}-n_{i} \equiv S
$$

Since the system is assumed to be periodic, one can Fourier decompose Eq.(6); as a result each Fourier mode associated with the electrostatic potential is given by

$$
\Phi_{k}=-\frac{S_{k}}{k^{2}}
$$

Clearly the long-wavelength modes in $S_{k}$ are 'amplified' in the electrostatic potential through the factor $k^{-2} \gg 1$. Therefore, it would be advantageous to resolve these modes accurately. At the other end of the $\mathbf{k}$ spectrum, the short-wavelength modes of the electrostatic potential generate strong 'random kicks' in the marker positions through the (dominant) $\mathbf{E} \times \mathbf{B}$ drift velocity; such short-wavelength modes can be partially suppressed by using, for example, a digital filter. The 'noise' is actually generated at all scale lengths and fed back into the simulation; as a result, the total energy of the gyrokinetic system is not conserved. Therefore, it should be advantageous to load the position of each ion guiding center such as to minimize the long wavelength modes in $\bar{n}_{i}$. Note that a simple random loading of the ion guiding centers is not optimal because of the convolution between configuration space and velocity space [see Eq.(5)].

Returning to our one-dimensional problem, the weight (which is related to the perturbed ion distribution function; see, for example, Ref. [4]) for marker $k$ is denoted $W_{k}$ and its position is $x_{k}$. A uniform grid in configuration space is setup where the node positions are given by $X_{g}=g \Delta x$, where $g=0,1, \cdots, N_{g}, \Delta x=L / N_{g}$ is the grid spacing and $N_{g}$ is the number of grid points. In view of the periodicity of the system, we have, for any physical quantity $F(x)$, the 
relation of $F(x+L)=F(x)$; for the discrete system, we have $F_{N_{g}}=F_{0}, F_{N_{g}+1}=F_{1}$, etc. where the notation $F_{g}=F\left(X_{g}\right)$ has been used. In order to be specific, we assume that the marker weights are loaded randomly according to

$$
W_{k}=\left(r_{k}-\frac{1}{2}\right) W_{0},
$$

where $W_{0} \ll 1$ is a small parameter and $r_{k}$ is a random number uniformly distributed in the interval $[0,1]$. We would like to point out that the algorithm described in this paper does not rely on Eq.(7) and it is valid for an arbitrary sequence $\mathbf{W}=\left\{W_{1}, W_{2}, \cdots, W_{N}\right\}$, where $N$ is the total number of markers used in the simulation. If $\rho_{g}$ denotes the number density at grid point $g$, we define an energy-like quantity of the form

$$
E(\mathbf{x}) \equiv E\left(x_{1}, x_{2}, \cdots, x_{N}\right) \equiv \sum_{g=1}^{N_{g}} \rho_{g}^{2}
$$

where, for each marker $k, x_{k}=x_{k}(t)$. In order to determine the number density at each node, we use the linear interpolation scheme to deposit the contribution of each marker onto the grid (Figure 1). We denote $\mathcal{D}\left(x_{k}, W_{k}\right)$ as the (linear) deposition scheme for marker $k$ with position $x_{k}$ and weight $W_{k}$. The algorithmic form of $\mathcal{D}\left(x_{k}, W_{k}\right)$ is

- find the grid index $g$ such that $g \Delta x \leq x_{k}<(g+1) \Delta x$;

- compute $\xi_{k}=\left(x_{k}-g \Delta x\right) / \Delta x=x_{k} / \Delta x-g$;

- update the (local) number density according to

$$
\begin{aligned}
\rho_{g} & \Longleftarrow \rho_{g}+\left(1-\xi_{k}\right) W_{k}, \\
\rho_{g+1} & \Longleftarrow \rho_{g+1}+\xi_{k} W_{k} .
\end{aligned}
$$

Here the left arrow denotes replacement. The updating rules for the marker position $x_{k}$ can be determined using Eq.(8)

$$
\begin{aligned}
\frac{d x_{k}}{d t} & =-\frac{\partial E}{\partial x_{k}} \\
& =-2 \sum_{g=1}^{N_{g}} \rho_{g} \frac{\partial \rho_{g}}{\partial x_{k}} \\
& =-2 \sum_{g=1}^{N_{g}} \sum_{k^{\prime}=1}^{N} \rho_{g} \frac{\partial \rho_{g}}{\partial x_{k^{\prime}}} \frac{\partial x_{k^{\prime}}}{\partial x_{k}} \\
& =\frac{2}{\Delta x}\left(\rho_{j}-\rho_{j+1}\right) W_{k}
\end{aligned}
$$

where the marker $k$ is assumed to belong to the cell $j$, that is $j \Delta x \leq x_{k}<(j+1) \Delta x$; the relation $\partial x_{k^{\prime}} / \partial x_{k}=\delta_{k k^{\prime}}$, where $\delta_{k k^{\prime}}$ is the Kronecker delta symbol, has also been used. Note that the dynamical equations (9) are coupled through the discrete number density. Eqs.(9) are integrated in time using standard fourth-order Runge-Kutta scheme [7]. The time step $\Delta t$ of integration is chosen such that the average displacement of the markers does not exceed the grid spacing. The energy-like quantity E, defined in Eq.(8), is monitored as the simulation progresses. The 
parameters used in Figures 2,3 and 4 are: $N_{g}=64, \Delta t=0.1$ and $N_{c}=N / N_{g}=50$ is the number of markers per cell.

Figure 2 shows the initial (plain line) number density based on a random initialization of the marker positions:

$$
x_{k}(0)=r_{k} L \quad(k=1,2, \cdots, N)
$$

where, as before, $r_{k}$ is a random number in the unit interval $[0,1]$. The dotted line shows the corresponding number density at $t=200$. Some residual short wavelength modes can be seen. Figure 3 shows the number density at $t=500$; we note the appearance of a long wavelength $(k \sim 1 / L)$ mode. The minima of the energy-like quantity $E$ is reached at saturation time $t=t_{s}$ such that

$$
\frac{d E}{d t}=0 \text { for } t \geq t_{s}
$$

The saturated number density profile is shown in Figure 4. The long wavelength mode, that was already visible in Figure 3, now dominates. Upon inspection of Figure 4 one can infer that the Fourier spectrum of the electrostatic potential is also dominated by a few long wavelength modes which are detrimental to the overall energy conservation. In comparison, the Fourier spectrum for the case of random loading (plain line) contained both short-wavelength and long-wavelength modes; the long-wavelength modes have relatively large amplitudes.

Figures 5 and 6 show the energy-like quantity as a function of time for the cases of $N_{c}=10$ markers per cell and $N_{c}=50$ markers per cell, respectively. Other parameters and the initial conditions are the same in Figures 2-4. In both cases, a steep initial decrease in $E$ can be seen. This is followed by a slower rate $d E / d t$; discrete events occur when a small group marker (or a single marker) cross over to a neighboring cell. Comparing Figure 5 with Figure 6 we note that the ratio $E\left(t_{s}\right) / E(0)$ is smaller for the case with $N_{c}=50$ markers per cell compared to the case with $N_{c}=10$. This is due to the fact that the random loading becomes a better approximation of the optimal loading as the number of markers $N$ is increased. In the limit $N \mapsto \infty$ the initial random loading is, of course, optimal.

\section{Two-dimensional Optimization Algorithm}

In this section, we describe a two-dimensional version of the algorithm presented in the previous section. The generalization of the present algorithm to toroidal geometry only requires the computation of new coefficients for the gyroaveraging processes (to account for the tilt in the magnetic field direction and the nonuniformity of the Jacobian).

The main difference with the case described in the previous section is that the number density of the ion guiding center, Eq.(5), now couples the configuration space to the velocity space. Furthermore, the gyroaveraging process (Figure 7) implies that the updating rules are nonlocal.

As it is customary in PIC simulations, one uses the magnetic moment per unit of mass, $\mu \equiv V_{\perp}^{2} /(2 B)$, instead of the perpendicular velocity as a dynamic variable. A Maxwellian probability distribution function is used for the loading in $V_{\perp}$

$$
F_{M}\left(V_{\perp}\right)=n_{0} \frac{V_{\perp}}{V_{t h i}^{2}} \exp \left(-\frac{V_{\perp}^{2}}{2 V_{\mathrm{thi}}{ }^{2}}\right) d V_{\perp},
$$

where $V_{\mathrm{thi}}=\sqrt{T_{\mathrm{i}} / m_{\mathrm{i}}}$ is the ion thermal velocity and $n_{0}$ is the equilibrium background density. The marker weights are loaded randomly, as in Eq.(7), although an arbitrary loading can be used. 
For simplicity, we consider a two-dimensional domain $\Omega=\left\{x \in\left[0, L_{x}\right], y \in\left[0, L_{y}\right]\right\}$ periodic in the $x$ and $y$ directions. In order to mimic the nonuniformity of the magnetic field in toroidal geometry, the confining $\mathbf{B}$ field is taken as

$$
\mathbf{B}=B_{0} \bar{x} / L_{s} \widehat{\mathbf{y}}+B_{0}[1-\epsilon \bar{x} \cos (2 \pi \bar{y})] \widehat{\mathbf{z}}
$$

where $L_{s}$ is the shear length, $(\bar{x}, \bar{y})=\left(x / L_{x}, y / L_{y}\right), \widehat{\mathbf{z}}$ is a unit vector in the $z$ direction and $\epsilon<1$ is a free parameter. The magnetic field given by Eq.(11) is obviously divergence free. A uniform computational grid with grid spacings $\Delta x=L_{x} / N_{x}$ and $\Delta y=L_{y} / N_{y}$ is set up. In analogy with the one-dimensional case, the energy-like quantity $E$ is defined as

$$
E=C \sum_{i=1}^{N_{x}} \sum_{j=1}^{N_{y}} \bar{n}_{i, j}^{2}(t),
$$

where $C=1 / \sum_{i=1}^{N_{x}} \sum_{j=1}^{N_{y}} \bar{n}_{i, j}^{2}(t=0)$ is a constant of normalization. If $\left(x_{k}, y_{k}\right)$ denotes the position of the guiding center of the $k^{\text {th }}$ marker, the locations of a set of $N_{g}$ gyropoints is defined through

$$
\begin{aligned}
& x_{k g}=x_{k}+\rho_{k} \cos \varphi_{g}, \\
& y_{k g}=y_{k}+\rho_{k} \sin \varphi_{g},
\end{aligned}
$$

for $g=1,2, \cdots, N_{g}$. In normalized gyrokinetic units, the magnitude of the gyroradius depends on the position in configuration space and velocity space since $\rho_{k}=V_{\perp k} / B\left(x_{k}, y_{k}\right)=$ $\sqrt{\mu_{k}} \sqrt{2 / B\left(x_{k}, y_{k}\right)}$. In Eq.(13), the discrete gyroangles are given by $\varphi_{g}=g \Delta \varphi$ and $\Delta \varphi=2 \pi / N_{g}$. The computation of the number density of ion guiding marker density is depicted in Figure 7 for the case $N_{g}=8$. The black square denotes the position of the guiding center whereas each small black triangle identifies a point on the ring with radius $\rho$, or simply a gyropoint. The small black circles denote the nodes of the computational grid; the cell $(i, j)$ denotes the area delimited by a set of four neighboring nodes: $(i, j),(i+1, j),(i, j+1)$ and $(i+1, j+1)$. The guiding center density at node $(i, j)$, which is denoted $\bar{n}_{i, j}$, usually involves the guiding centers of markers which belong to different cells. In order words, a change in the marker position $\left(x_{k}, y_{k}\right) \mapsto\left(x_{k}+\delta x_{k}, y_{k}+\delta y_{k}\right)$ will affect the number density at different nodes $(i, j)$. The two-dimensional version of the linear interpolation scheme is used (See Figure 8). If the position of the gyropoint $\left(x_{k g}, y_{k g}\right)$ satisfies the relations of

$$
\begin{aligned}
& i \Delta x \leq x_{k g}<(i+1) \Delta x, \\
& j \Delta y \leq y_{k g}<(j+1) \Delta y,
\end{aligned}
$$

then its contribution to the local number density is

$$
\begin{aligned}
\bar{n}_{i, j} & \Longleftarrow \bar{n}_{i, j}+\sigma_{00} \frac{W_{k}}{N_{g}}, \\
\bar{n}_{i+1, j} & \Longleftarrow \bar{n}_{i+1, j}+\sigma_{10} \frac{W_{k}}{N_{g}}, \\
\bar{n}_{i, j+1} & \Longleftarrow \bar{n}_{i, j+1}+\sigma_{01} \frac{W_{k}}{N_{g}}, \\
\bar{n}_{i+1, j+1} & \Longleftarrow \bar{n}_{i+1, j+1}+\sigma_{11} \frac{W_{k}}{N_{g}}
\end{aligned}
$$


where $\sigma_{11}=\alpha \beta, \sigma_{10}=\alpha(1-\beta), \sigma_{01}=\beta(1-\alpha), \sigma_{00}=(1-\alpha)(1-\beta), \alpha=x_{k g} / \Delta x-i$ and $\beta=y_{k g} / \Delta y-j$. In analogy with the one-dimensional case, the updating rules for the guiding center position are

$$
\begin{aligned}
& \frac{d x_{k}}{d t}=-\frac{\partial E}{\partial x_{k}} \\
& \frac{d y_{k}}{d t}=-\frac{\partial E}{\partial y_{k}}
\end{aligned}
$$

where $E$ is given by Eq.(12). At $t=0$ the markers are loaded randomly in the domain $\Omega$. Figure 9 shows the time evolution of the energy-like quantity as a function of time. The simulation parameters are $N_{c}=N / N_{g}=10, N_{x}=N_{y}=64, L_{x}=L_{y}=8, \Delta t=1.0$ and $N_{g}=4$. Figure 9 shows that $E(t)$ follows a pattern similar to those of Figures 5 and 6 . However, the discrete events that occur in Figures 5 and 6 are absent in Figure 9. This smooth behavior is due to the gyroaveraging process which makes the updating rules (14) nonlocal. For example, the guiding center shown in Figure 7 influences the local number density at no less than 16 nodes.

Figure 10 shows the initial density of ion guiding centers. For this specific case, the updating rules (14) involve a set of $2 N_{x} N_{y} N_{c}=81920$ coupled equations. The local density of ion guiding centers at $t=100$ is shown in Figure 11. As in the one-dimensional case, the shortwavelength modes are gradually removed from the simulation domain although a few modes with $k_{\perp} \Delta x \sim k_{\perp} \Delta y=\mathcal{O}(1)$ do remain; these long-wavelength modes have small amplitudes. Figure 12 shows the final (saturated) number density profile. The amplitudes of the dominant modes is roughly two orders of magnitude smaller than those of Figure 10. Further, the Fourier spectrum is, as in the one-dimensional case, dominated by a few long-wavelength modes.

We now compare the energy conservation properties associated with the optimized loading algorithm. The marker weights are advanced in phase space using the $\delta f$ algorithm along their respective trajectories (the derivation of the basic equations can be found elsewhere [8] and are not repeated here). As shown in the Appendix, the gyrokinetic system should conserve the total energy defined as

$$
\frac{E_{\mathrm{tot}}}{n_{0} T_{e}}=\frac{K(t)}{n_{0} T_{e}}+\frac{U(t)}{n_{0} T_{e}}
$$

where

$$
\frac{U(t)}{n_{0} T_{e}}=\frac{1}{2} \int\left|\nabla_{\perp} \Phi\right|^{2} d^{3} x
$$

is the potential energy and

$$
\frac{K(t)}{n_{0} T_{e}}=\iint F \frac{v^{2}}{2} d^{3} x d^{3} v,
$$

is the kinetic energy. The right-hand sides of Eqs. $(15,16)$ are in gyrokinetic units. In order to measure the nonconservation of total energy, the quantity

$$
\Delta E(t) \equiv \frac{\left|E_{\mathrm{tot}}(t)-E_{\mathrm{tot}}(0)\right|}{n_{0} T_{e} L_{x} L_{y}},
$$

has been monitored throughout the simulation. Figure 13 shows that the nonconversation of energy becomes substantial for $t \geq 4000$. However, $\Delta E$ increases much faster when a random 
loading (plain line) is used as compared to the case of an optimized loading (dotted line). Furthermore, as the end of the simulation $\Delta E$ is roughly of an order of magnitude larger for the random loading case than when compared to the optimized loading case. This difference in the energy conservation properties can be traced to the 'random kicks' experienced by the markers at $t=0$. Each marker has a 'memory' of its trajectory through phase space; the cumulative effect of an inaccurate initial loading translates into poor energy conservation in the late nonlinear stage of the simulation.

\section{Conclusions}

The problem of marker loading in particle-in-cell gyrokinetic simulations has been addressed using a quadratic optimization algorithm. It has been shown that optimized loading in configuration space dramatically reduces the short wavelength modes of the electrostatic potential which are partly responsible for the nonconservation of total energy. As the same time, the long wavelength modes in the ion guiding center density (that tend to be amplified in the electrostatic potential) are resolved with accuracy. It is this combination of factors (damping of short-wavelength modes and better resolution of long-wavelength modes) which results in better overall energy conservation. The method presented in this paper can be easily extended to arbitrary toroidal plasmas. Although the computing power of massively-parallel supercomputers has increased considerably over the past decade, full-torus, gyrokinetic PIC simulations of microturbulence in tokamak plasmas typically operate with 5 to 15 markers per cell; we believe that the energy conservation properties of such simulated plasmas can be improved using optimized loading in configuration space and velocity space.

\section{Acknowledgments}

This research was supported by Contract No DE-AC02-76CH03073 and the Scientific Discovery through Advanced Computing (SciDAC) initiative (U.S. Department of Energy). 


\section{Appendix: Energy conservation in electrostatic gyroki- netic simulations}

The guiding center distribution for particle species $j$ in the electrostatic, collisionless limit is governed by

$$
\frac{\partial F_{j}}{\partial t}+\nabla \cdot\left(F_{j} \frac{d \mathbf{R}}{d t}\right)+\frac{\partial}{\partial v_{\|}}\left(F_{j} \frac{d v_{\|}}{d t}\right)=0
$$

where the equations of motions are

$$
\frac{d \mathbf{R}}{d t}=v_{\|} \widehat{\mathbf{b}}^{\star}+\frac{\mu}{\omega_{c j}} \widehat{\mathbf{b}} \times \nabla B+\mathbf{V}_{E}
$$

and

$$
\frac{d v_{\|}}{d t}=-\widehat{\mathbf{b}}^{\star} \cdot\left(\mu \nabla B+\frac{q_{j}}{m_{j}} \nabla \Phi\right)
$$

and $\mathbf{V}_{E}=c \widehat{\mathbf{b}} \times \nabla \Phi / B, \widehat{\mathbf{b}^{\star}} \equiv \widehat{\mathbf{b}}+\rho_{\|} \widehat{\mathbf{b}} \times \boldsymbol{\kappa}, \rho_{\|}=v_{\|} / \omega_{c j}$ is the parallel gyroradius and $\mu=v_{\perp}^{2} /(2 B)$ is the magnetic moment. Let us operate with $\int v^{2} / 2(\bullet) d^{3} x d^{3} v$ on Eq.(18):

$$
\underbrace{\frac{\partial}{\partial t} \int F_{j} \frac{v^{2}}{2} d^{3} x d^{3} v}_{1}+\underbrace{\int \boldsymbol{\nabla} \cdot\left(\frac{v^{2}}{2} F_{j} \frac{d \mathbf{R}}{d t}\right) d^{3} x d^{3} v}_{2}+\underbrace{\int \frac{v^{2}}{2} \frac{\partial}{\partial v_{\|}}\left(F_{j} \frac{d v_{\|}}{d t}\right) d^{3} x d^{3} v}_{3}=0 .
$$

Term 2 in the above equation vanishes whereas term 3 can be integrated by parts; as a result we obtain

$$
\frac{\partial}{\partial t} \int F_{j} \frac{v^{2}}{2} d^{3} x d^{3} v=\int F_{j} v_{\|} \frac{d v_{\|}}{d t} d^{3} x d^{3} v
$$

Eq.(19) can be written as

$$
v_{\|} \widehat{\mathbf{b}}^{\star}=\frac{d \mathbf{R}}{d t}-\frac{\mu}{\omega_{c j}} \widehat{\mathbf{b}} \times \nabla B-\mathbf{V}_{E} .
$$

Multiplying Eq.(20) by $v_{\|}$and using Eq.(23), one gets

$$
\begin{aligned}
v_{\|} \frac{d v_{\|}}{d t} & =\underbrace{\frac{\mu^{2}}{\omega_{c j}}(\widehat{\mathbf{b}} \times \nabla B) \cdot \boldsymbol{\nabla} B}_{1}+\underbrace{\mu \frac{q_{j}}{m_{j} \omega_{c j}}(\widehat{\mathbf{b}} \times \nabla B) \cdot \nabla \Phi}_{2} \\
& +\underbrace{\mu \mathbf{V}_{E} \cdot \boldsymbol{\nabla} B}_{3}+\underbrace{\frac{q_{j}}{m_{j}} \mathbf{V}_{E} \cdot \boldsymbol{\nabla} \Phi}_{4}-\underbrace{\mu \boldsymbol{\nabla} B \cdot \frac{d \mathbf{R}}{d t}}_{5}-\underbrace{\frac{q_{j}}{m_{j}} \boldsymbol{\nabla} \Phi \cdot \frac{d \mathbf{R}}{d t}}_{6}
\end{aligned}
$$

where terms 1 and 4, as well as the sum of terms 2 and 3, vanish. Eq.(24) then simplifies to

$$
v_{\|} \frac{d v_{\|}}{d t}=-\frac{d \mathbf{R}}{d t} \cdot\left(\mu \nabla B+\frac{q_{j}}{m_{j}} \nabla \Phi\right) .
$$


Using the relation of

$$
\int \boldsymbol{\nabla} \cdot\left(F_{j} \frac{d \mathbf{R}}{d t} \Phi\right) d^{3} x=\int F_{j} \frac{d \mathbf{R}}{d t} \cdot \nabla \Phi d^{3} x+\int \Phi \nabla \cdot\left(F_{j} \frac{d \mathbf{R}}{d t}\right) d^{3} x=0,
$$

it follows that

$$
\iint F_{j} \frac{d \mathbf{R}}{d t} \cdot \nabla \Phi d^{3} x d^{3} v=-\iint \Phi \nabla \cdot\left(F_{j} \frac{d \mathbf{R}}{d t}\right) d^{3} x d^{3} v .
$$

Using the expression for the volume element in velocity space, $d^{3} v=B d \mu d v_{\|}$, we note that

$$
\begin{aligned}
\iint \mu F_{j} \nabla B \cdot \frac{d \mathbf{R}}{d t} d^{3} x d^{3} v & =\iint \mu F_{j} \boldsymbol{\nabla}\left(\frac{B^{2}}{2}\right) \cdot \frac{d \mathbf{R}}{d t} d \mu d v_{\|} d^{3} x \\
& =\iint \mu \nabla \cdot\left(F_{j} \frac{B^{2}}{2} \frac{d \mathbf{R}}{d t}\right) d \mu d v_{\|} d^{3} x \\
& -\iint \frac{\mu B^{2}}{2} \nabla \cdot\left(F_{j} \frac{d \mathbf{R}}{d t}\right) d \mu d v_{\|} d^{3} x \\
& =-\iint \frac{\mu B}{2} \nabla \cdot\left(F_{j} \frac{d \mathbf{R}}{d t}\right) d^{3} x d^{3} v
\end{aligned}
$$

Using Eqs.(26,27) in Eq.(22), one gets

$$
\frac{\partial}{\partial t}=\iint F_{j} \frac{v^{2}}{2} d^{3} x d^{3} v=\iint\left(\frac{q_{j} \Phi}{m_{j}}+\frac{\mu B}{2}\right) \nabla \cdot\left(F_{j} \frac{d \mathbf{R}}{d t}\right) d^{3} x d^{3} v .
$$

Using the relation of

$$
\boldsymbol{\nabla} \cdot\left(F_{j} \frac{d \mathbf{R}}{d t}\right)=-\frac{\partial F_{j}}{\partial t}-\frac{\partial}{\partial v_{\|}}\left(F_{j} \frac{d v_{\|}}{d t}\right)
$$

in Eq.(28) we obtain

$$
\frac{\partial}{\partial t} \iint F_{j} \frac{v^{2}}{2} d^{3} x d^{3} v=-\frac{q_{j}}{m_{j}} \int \Phi \frac{\partial n_{j}}{\partial t} d^{3} x
$$

where $n_{j}=\int F_{j} d^{3} v$ is the number density for particle species $j$. To proceed further, we use the gyrokinetic Poisson equation (in the long wavelength limit)

$$
n_{0} \rho_{s}^{2} \frac{e}{T_{e}} \nabla_{\perp}^{2} \Phi=n_{e}-n_{i}
$$

Upon multiplication of Eq.(29) by $m_{j}$ and summation over species, and taking into account Eq.(30), one obtains

$$
\frac{\partial}{\partial t} \sum_{j} m_{j} \iint F_{j} \frac{v^{2}}{2} d^{3} x d^{3} v=e^{2} \int n_{0} \frac{\rho_{s}^{2}}{T_{e}} \Phi \nabla_{\perp}^{2} \frac{\partial \Phi}{\partial t} d^{3} x
$$

Using the relation of

$$
\Phi \frac{\partial}{\partial t} \nabla_{\perp}^{2} \Phi=\nabla_{\perp} \cdot\left(\Phi \frac{\partial}{\partial t} \nabla_{\perp} \Phi\right)-\frac{1}{2} \frac{\partial}{\partial t}\left|\nabla_{\perp} \Phi\right|^{2}
$$

and neglecting the (slow) variation of $n_{0} \rho_{s}^{2} / T_{e}$ in Eq.(31) we obtain an equation describing the conservation of energy

$$
\sum_{j} \iint F_{j} \frac{m_{j} v^{2}}{2} d^{3} x d^{3} v+\frac{1}{8 \pi} \frac{\rho_{s}^{2}}{\lambda_{D}{ }^{2}} \int\left|\nabla_{\perp} \Phi\right|^{2} d^{3} x=E_{0}=\text { const },
$$

where $\lambda_{D}=\sqrt{T_{e} /\left(4 \pi e^{2} n_{0}\right)}$ is the Debye length. 


\section{References}

[1] P. C. Liewer, Nucl. Fusion 25, 543 (1985).

[2] F. Wagner and U. Stroth, Plasma Phys. Contr. Fusion, 35, 1321 (1993).

[3] W. Horton, Rev. Mod. Phys. 71, 735 (1999).

[4] G. Hu and J.A. Krommes, Phys. Plasmas, 1(4), 863 (1994).

[5] J.L.V. Lewandowski, Phys. Lett. A, 313, 291 (2003).

[6] A. Brizard, J. Plasma Phys. 41, 541 (1989).

[7] W.S Press, S.A. Teukolsky, W.T. Vetterling and B.P. Flannery, Numerical Recipes in Fortran (Cambridge University Press, New York, 1992).

[8] R.E. Denton and M. Kotschenreuther, J. Comp. Phys. 119, 283 (1995). 
Figure 1 One-dimensional, linear interpolation for the computation of the local number density. The vertical arrow shows the position of the $k^{\text {th }}$ marker (see main text for definition of $\xi_{k}$ ).

Figure 2 Histogram of the discrete number density for the initial loading (plain line) and for the optimized loading at $t=200$; the parameters of the simulations are: $N_{g}=64$ (number of nodes), $\Delta t=0.1$ (time step of integration) and $N_{c}=50$ (number of markers per cell).

Figure 3 Same as Figure 2 but at $t=500$.

Figure 4 Same as Figure 2 but at $t=2000$ (saturation).

Figure 5 Time evolution of the energy-like quantity $E$ for the case of $N_{c}=10$ markers per cell. Other parameters are: $N_{g}=64$ (number of nodes) and $\Delta t=0.1$.

Figure 6 Time evolution of the energy-like quantity $E$ for the case of $N_{c}=50$ markers per cell. Other parameters are the same as in Figure 5.

Figure 7 Computation of the number density of ion guiding centers. The position of a guiding center is shown by a square with coordinates $(X, Y)$. A set of $N_{g}=8$ of so-called gyropoints is also shown (triangles). The computational nodes (or grid points) are represented by filled circles.

Figure 8 Two-dimensional linear interpolation scheme.

Figure 9 Profile of the energy-like quantity $E$ as a function of time. The simulation parameters are: $N_{x}=N_{y}=64, N_{c}=N /\left(N_{x} N_{y}\right)=10, N_{g}=4, L_{x}=L_{y}=8$ and $\Delta t=1.0$. The initial marker positions are purely random at $t=0$.

Figure 10 Initial number density in the domain $\Omega=\left\{x \in\left[0, L_{x}\right], y \in\left[0, L_{y}\right]\right\}$.

Figure 11 Same as Figure 10 at $t=100$.

Figure 12 Same as Figure 10 at saturation $(t=4000)$.

Figure 13 Variation of the total energy for a two-dimensional gyrokinetic PIC simulations for a random initial loading (plain line) and an optimized loading (dotted line) 
Figure 1 Lewandowski

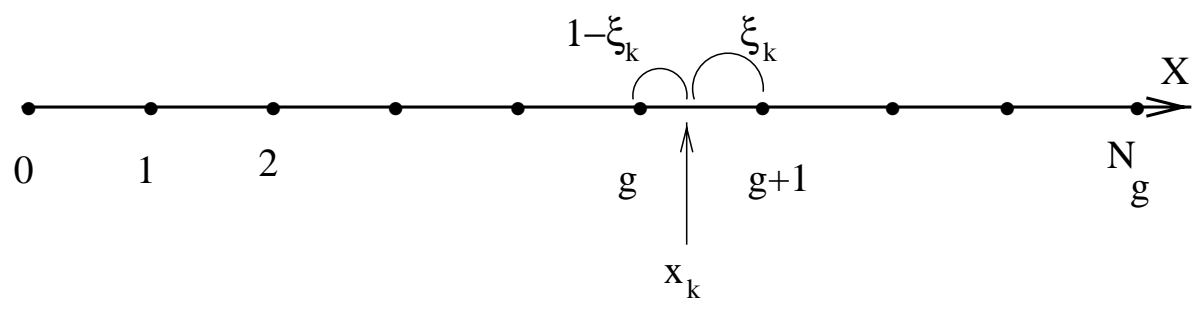


Figure 2 Lewandowski

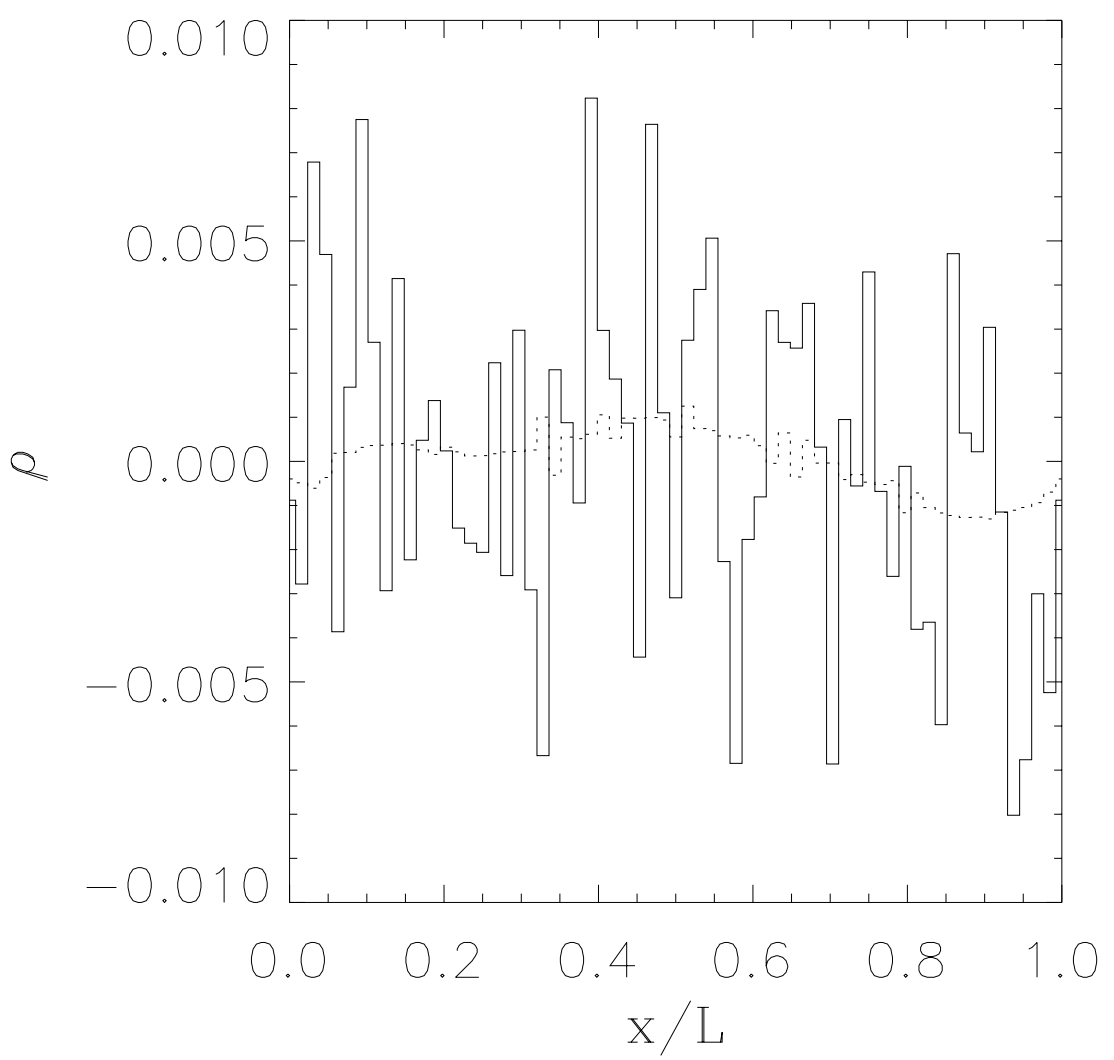


Figure 3 Lewandowski

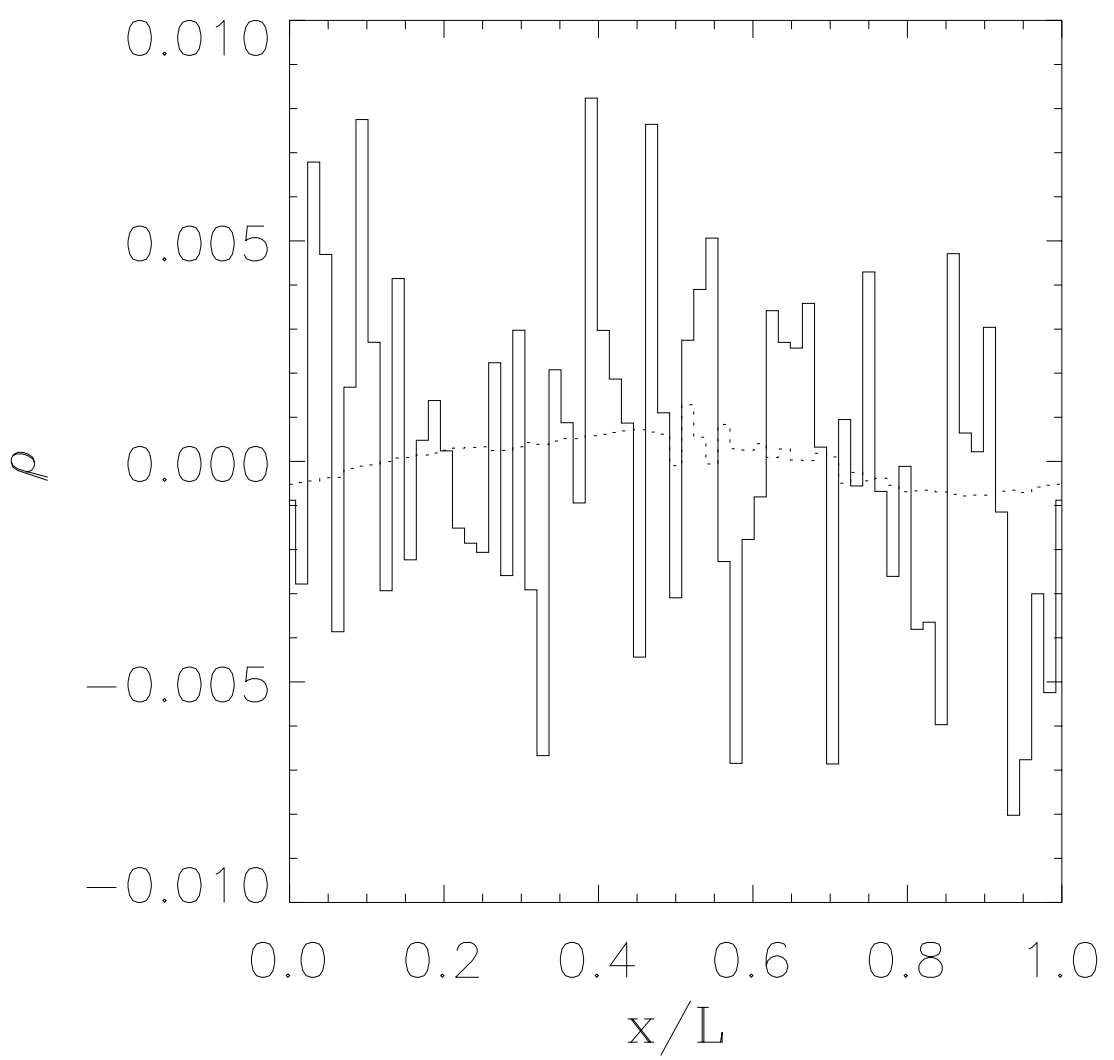


Figure 4 Lewandowski

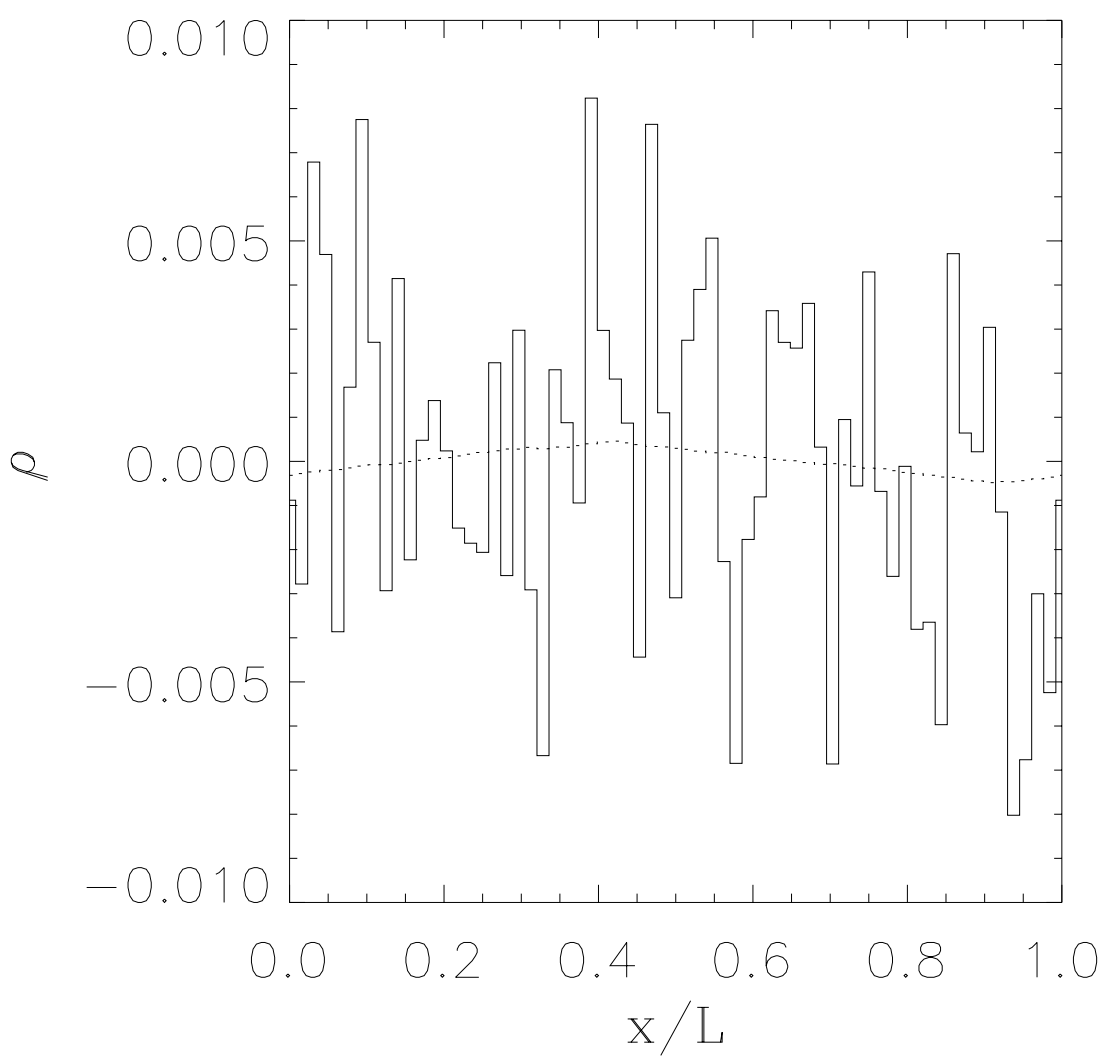


Figure 5 Lewandowski

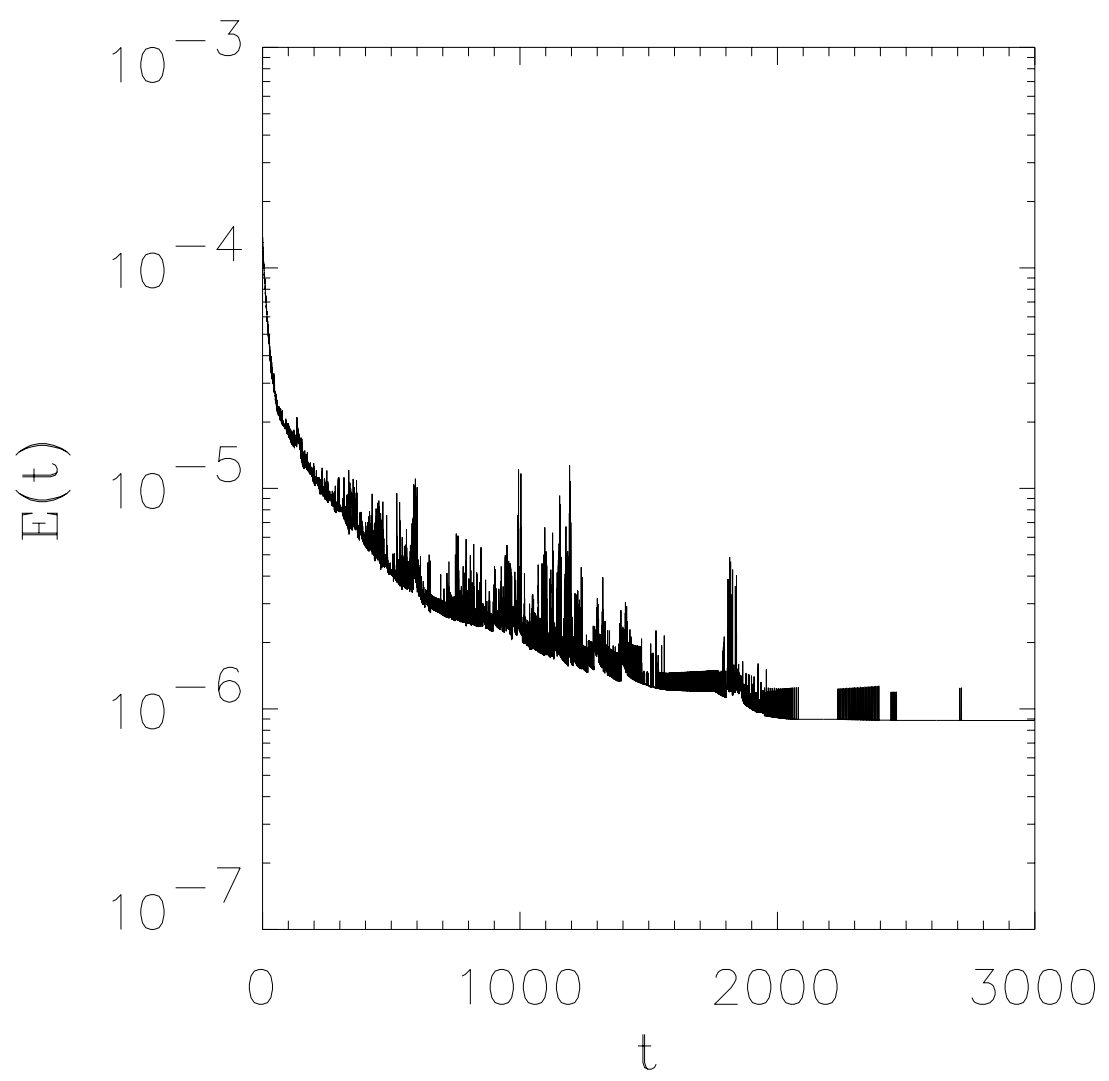


Figure 6 Lewandowski

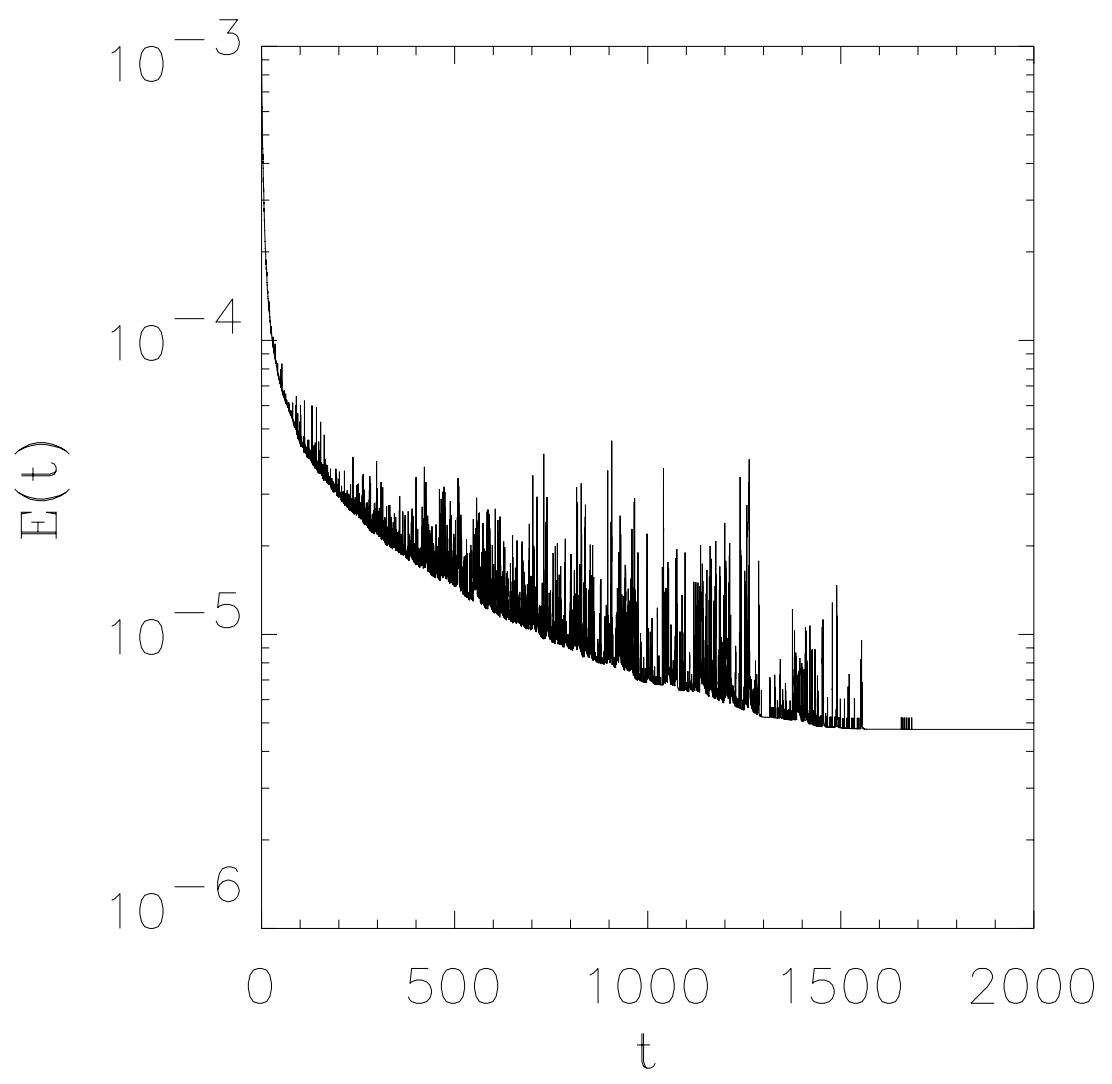


Figure 7 Lewandowski

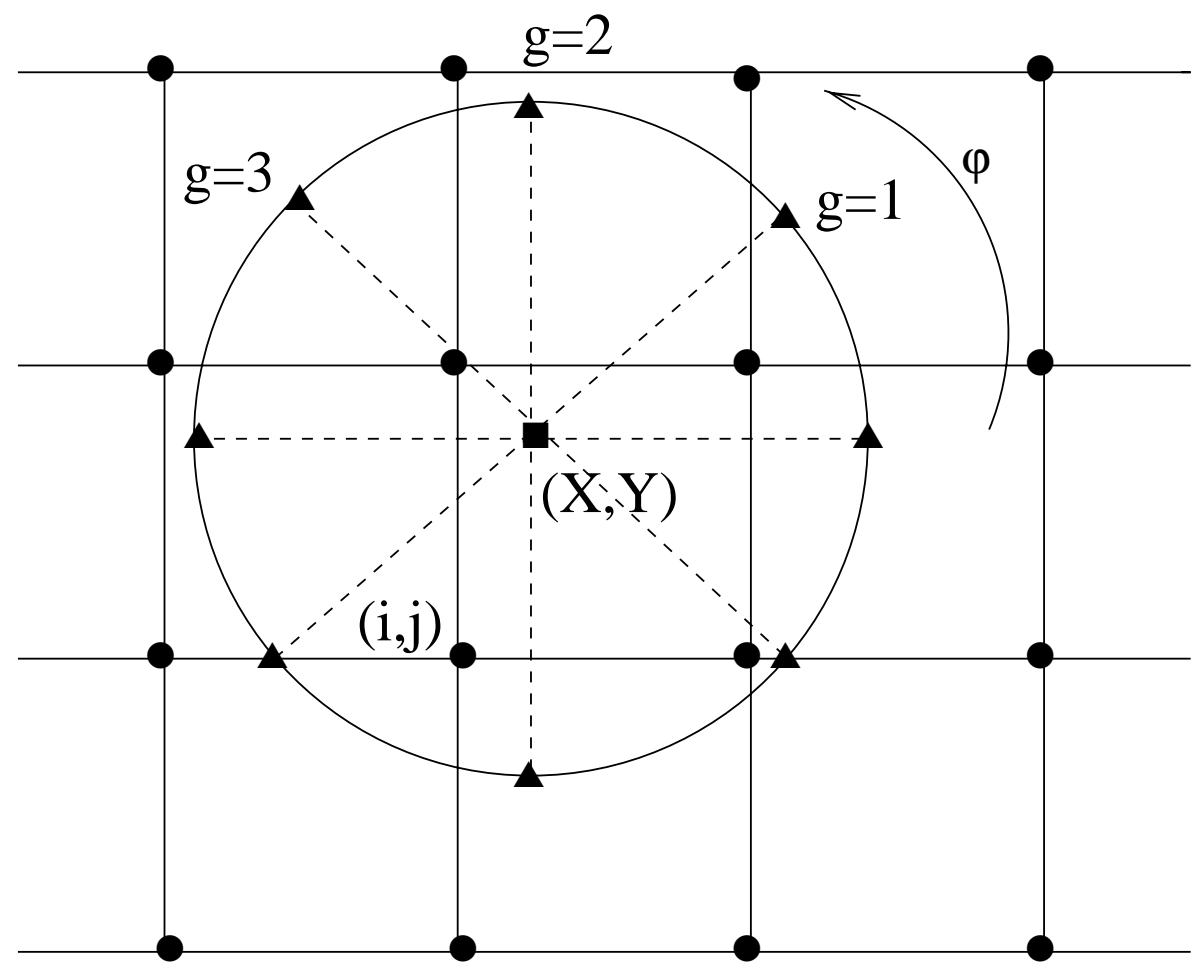


Figure 8 Lewandowski

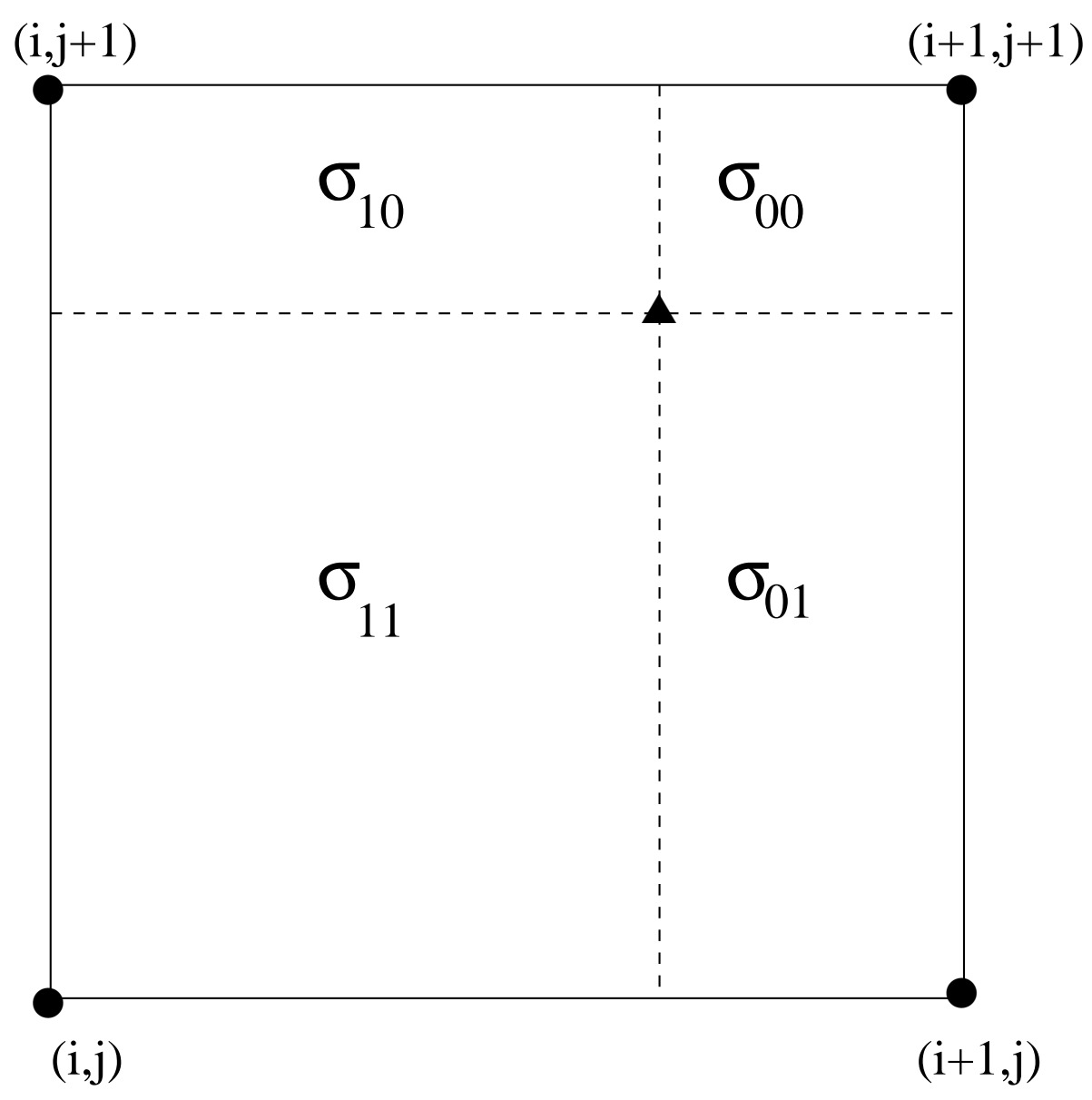


Figure 9 Lewandowski

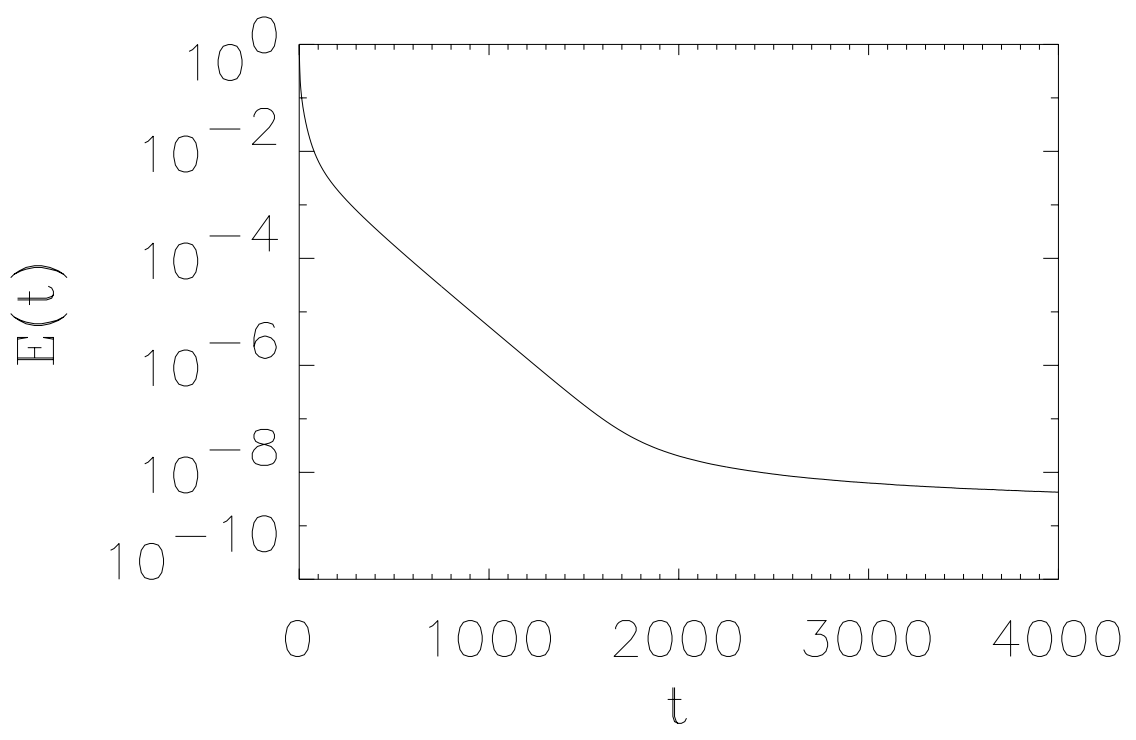




\section{Figure 10 Lewandowski}

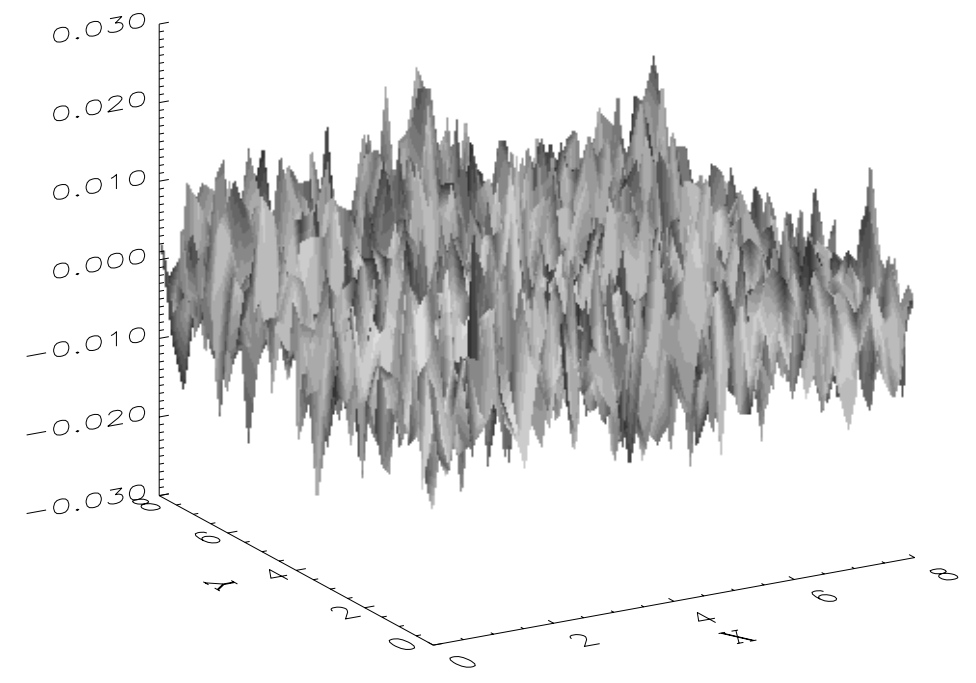


Figure 11 Lewandowski

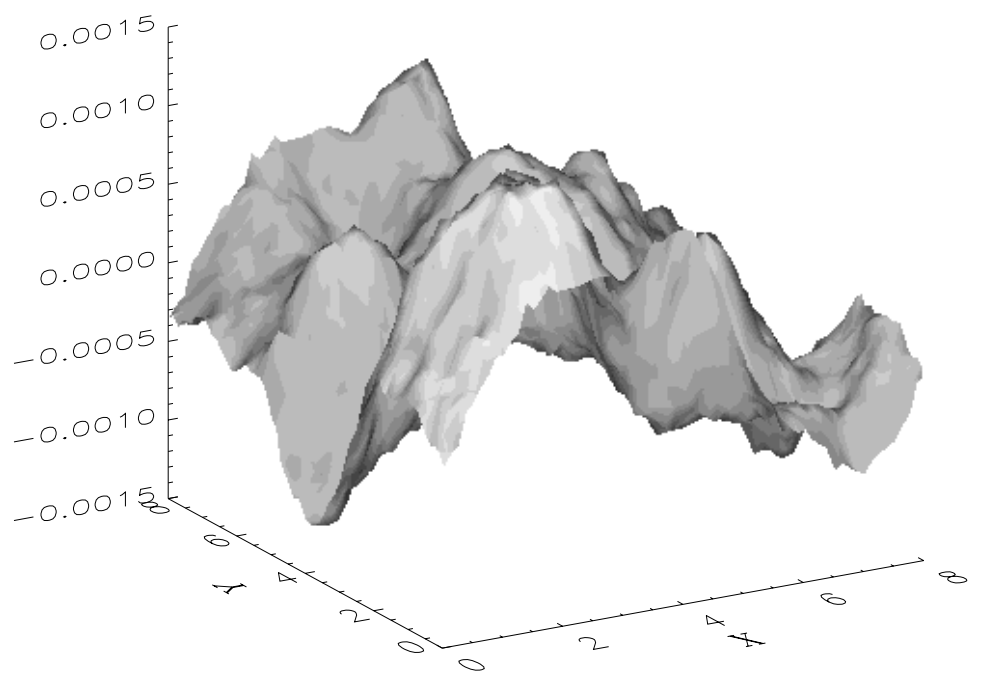


Figure 12 Lewandowski

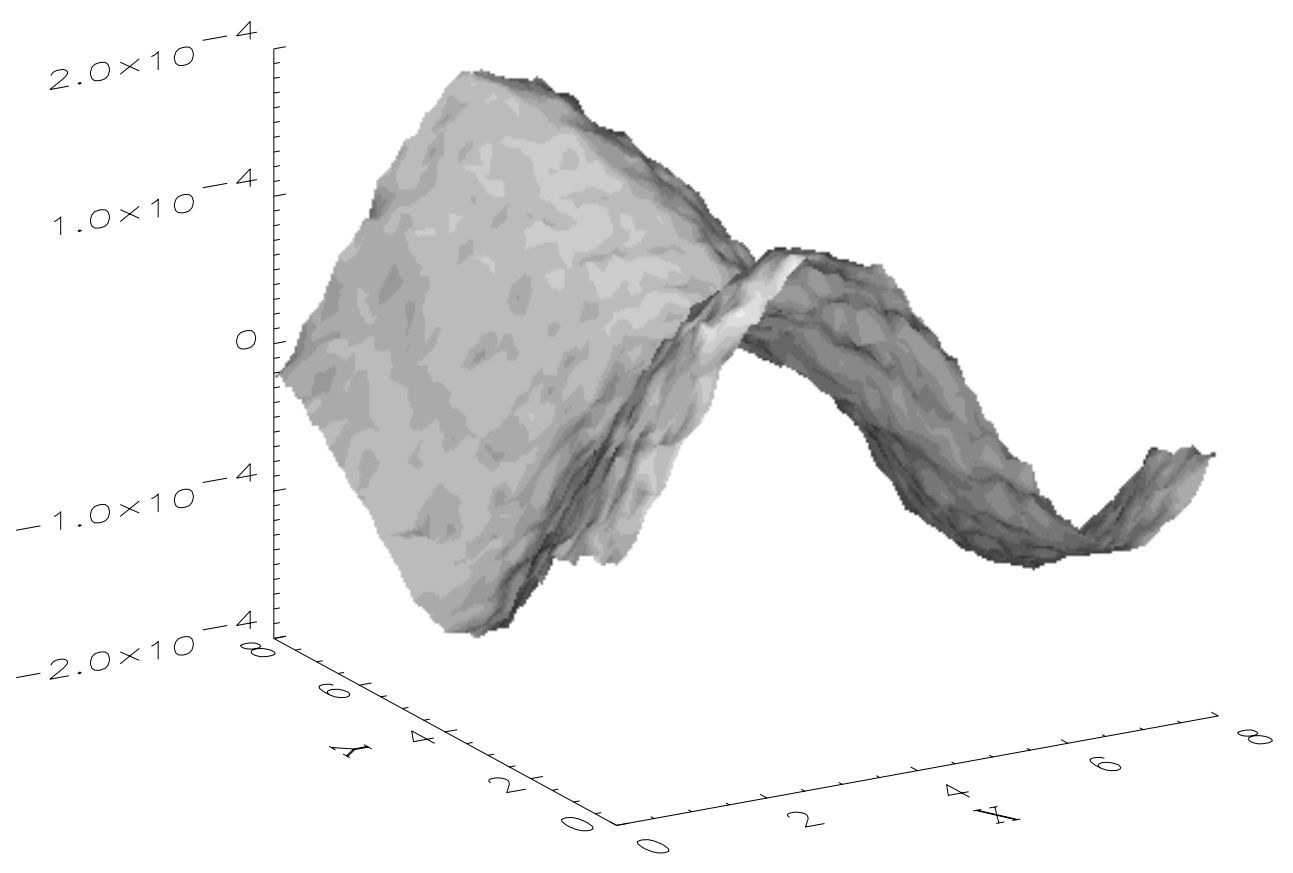


Figure 13 Lewandowski

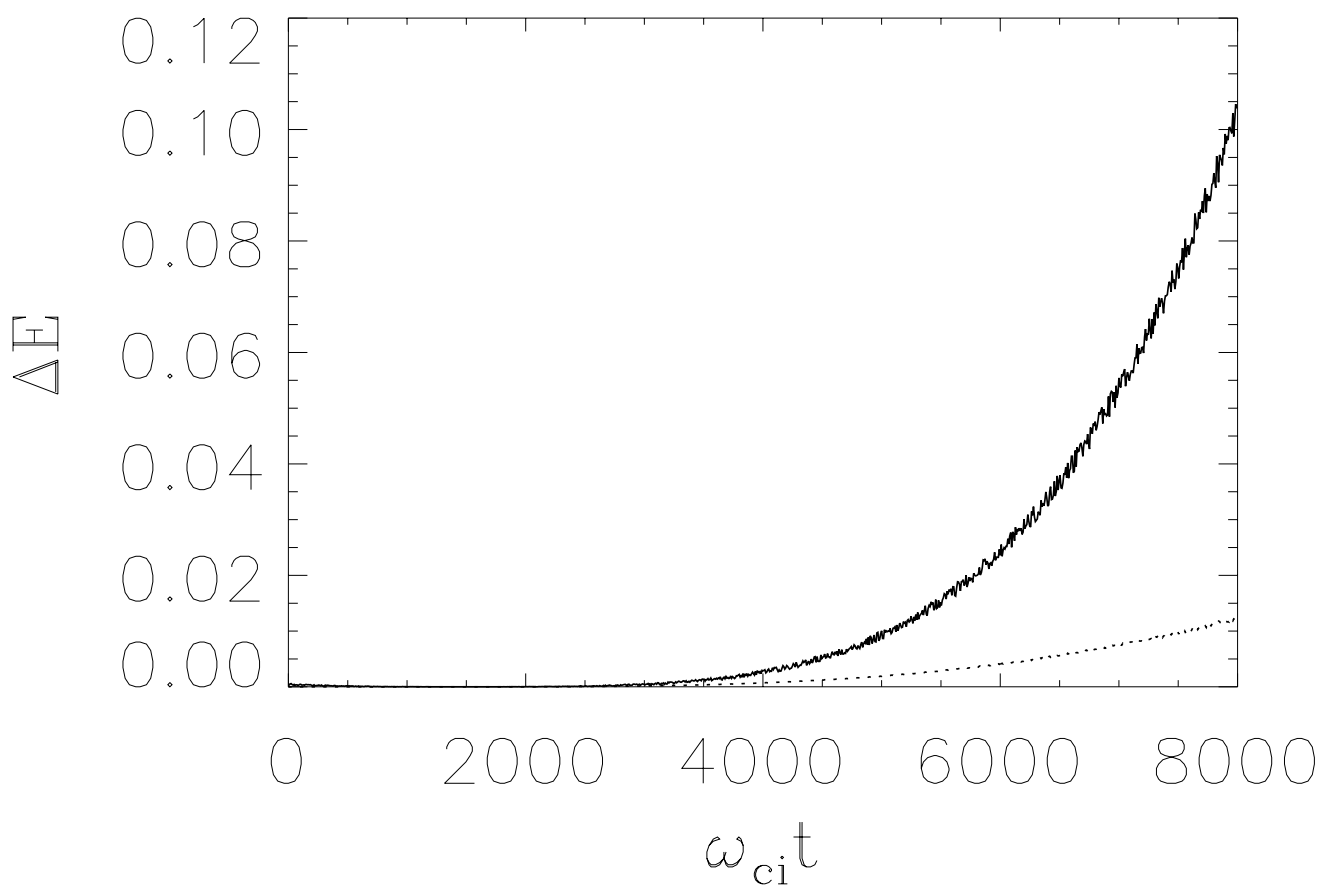




\section{External Distribution}

Plasma Research Laboratory, Australian National University, Australia

Professor I.R. Jones, Flinders University, Australia

Professor João Canalle, Instituto de Fisica DEQ/IF - UERJ, Brazil

Mr. Gerson O. Ludwig, Instituto Nacional de Pesquisas, Brazil

Dr. P.H. Sakanaka, Instituto Fisica, Brazil

The Librarian, Culham Laboratory, England

Mrs. S.A. Hutchinson, JET Library, England

Professor M.N. Bussac, Ecole Polytechnique, France

Librarian, Max-Planck-Institut für Plasmaphysik, Germany

Jolan Moldvai, Reports Library, Hungarian Academy of Sciences, Central Research Institute for Physics, Hungary

Dr. P. Kaw, Institute for Plasma Research, India

Ms. P.J. Pathak, Librarian, Institute for Plasma Research, India

Ms. Clelia De Palo, Associazione EURATOM-ENEA, Italy

Dr. G. Grosso, Instituto di Fisica del Plasma, Italy

Librarian, Naka Fusion Research Establishment, JAERI, Japan

Library, Laboratory for Complex Energy Processes, Institute for Advanced Study, Kyoto University, Japan

Research Information Center, National Institute for Fusion Science, Japan

Dr. O. Mitarai, Kyushu Tokai University, Japan

Dr. Jiangang Li, Institute of Plasma Physics, Chinese Academy of Sciences, People's Republic of China

Professor Yuping Huo, School of Physical Science and Technology, People's Republic of China

Library, Academia Sinica, Institute of Plasma Physics, People's Republic of China

Librarian, Institute of Physics, Chinese Academy of Sciences, People's Republic of China

Dr. S. Mirnov, TRINITI, Troitsk, Russian Federation, Russia

Dr. V.S. Strelkov, Kurchatov Institute, Russian Federation, Russia

Professor Peter Lukac, Katedra Fyziky Plazmy MFF UK, Mlynska dolina F-2, Komenskeho Univerzita, SK-842 15 Bratislava, Slovakia

Dr. G.S. Lee, Korea Basic Science Institute, South Korea

Institute for Plasma Research, University of Maryland, USA

Librarian, Fusion Energy Division, Oak Ridge National Laboratory, USA

Librarian, Institute of Fusion Studies, University of Texas, USA

Librarian, Magnetic Fusion Program, Lawrence Livermore National Laboratory, USA

Library, General Atomics, USA

Plasma Physics Group, Fusion Energy Research Program, University of California at San Diego, USA

Plasma Physics Library, Columbia University, USA

Alkesh Punjabi, Center for Fusion Research and Training, Hampton University, USA

Dr. W.M. Stacey, Fusion Research Center, Georgia Institute of Technology, USA

Dr. John Willis, U.S. Department of Energy, Office of Fusion Energy Sciences, USA

Mr. Paul H. Wright, Indianapolis, Indiana, USA 
The Princeton Plasma Physics Laboratory is operated by Princeton University under contract with the U.S. Department of Energy.

\author{
Information Services \\ Princeton Plasma Physics Laboratory \\ P.O. Box 451 \\ Princeton, NJ 08543
}

Phone: 609-243-2750

Fax: 609-243-2751

e-mail: pppl_info@pppl.gov

Internet Address: http://www.pppl.gov 